\title{
Emotions Detection using Java and Neural Networks
}

\author{
Ayeni Olaniyi Abiodun \\ Department of computer \\ science, Federal University of \\ Technology, Akure
}

\author{
Mogaji Stephen Alaba \\ Department of Computer \\ Studies, Federal Science and \\ Technical College \\ Ikare-Akoko, Ondo state \\ Nigeria
}

\author{
Olayemi Olufunke C. \\ Department of computer \\ science, Joseph-Ayo Babalola \\ University, Ikeji Arakeji
}

\begin{abstract}
This research work focuses on the design and implementation of a facial expression detection system focusing mainly on three expressions which are happiness, anger and sadness. Every image is received as jpeg files, Images are then preprocessed forl feature extractions. Feature extraction on each images received is extracted using principal component analysis (PCA), extracted features are passed to the neural networks. Java object oriented programming language and support vector machines (SVM) algorithm was used in the design and implementation of the system. In this research work, facial expression detecting system is implemented using java programming language and SVM algorithm. The project introduces a simple architecture for human facial expression detection and elaborate more on what facial expression and facial expression detection system is about. The project looks into the existing system, identifies the problem thereby deploying a new system which is user interactive. The system developed achieved a good result of $81.25 \%$ for happiness, $75 \%$ for anger and $60 \%$ for sadness.
\end{abstract}

\section{General Terms}

Facial Recognition, Security, Computer vision, machine learning

\section{Keywords}

Facial expression, images, emotions, detection, recognition, classification, java programming language and system

\section{INTRODUCTION}

Facial expression is one or more motions or positions of the muscles beneath the skin of the face.[29] These movements convey the emotional state of an individual to observers. Facial expressions are the most important information for emotions perception in face to face communication and a form of nonverbal communication. They are a primary means of conveying social information between humans, but they also occur in most other mammals and some other animal species. Facial expression detection is one of the most relevant applications of image processing and biometric system, it is not an easy task because of circumstances like illumination, facial occlusions, face colour, shape etc. These expressions can vary between individuals[28]. Face detection systems have many problems pertaining to pose, light, facial expression and quality of picture. It can be solved by applying some sort of image preprocessing before they are applied for further analysis purpose. Face detection determines the locations and sizes of faces in an input image, they are easily located in cluttered scenes by infants and adults alike. However, automatic human face detection by computers is a very challenging task because face patterns can have significantly variable image appearances[28]\&[29]. For example, human faces vary from genders, ages, hair styles and races etc. In addition, the variations of scales, shapes and poses of faces in images also hinder the success of automatic face detection systems. Several different approaches have been proposed to solve the problem of face detection and each approach has its own advantages and disadvantages[28].

\section{STATEMENT OF PROBLEM}

Emotions are read by humans through detection of face muscles and behaviour of some features on the face. We detect faces effortlessly in a wide range of conditions, under bad lightning conditions or from a great distance and this conditions might not allow us to get the accurate expression of the face. This system will help in reducing the difficulty in detecting human expressions accurately.

\section{THE PROPOSED SYSTEM}

Looking into the problem statement and having an insight on the already existing system, the system will be able to detect the primary emotions which are sadness, anger and happiness and to easily tell if the user is in any of this three states. The system will be human-computer interaction systems that will help detect human facial expression and emotions, it will provide the ability to perform real-time, frame-by-frame analysis of the emotional responses of users, detecting and tracking expressions.

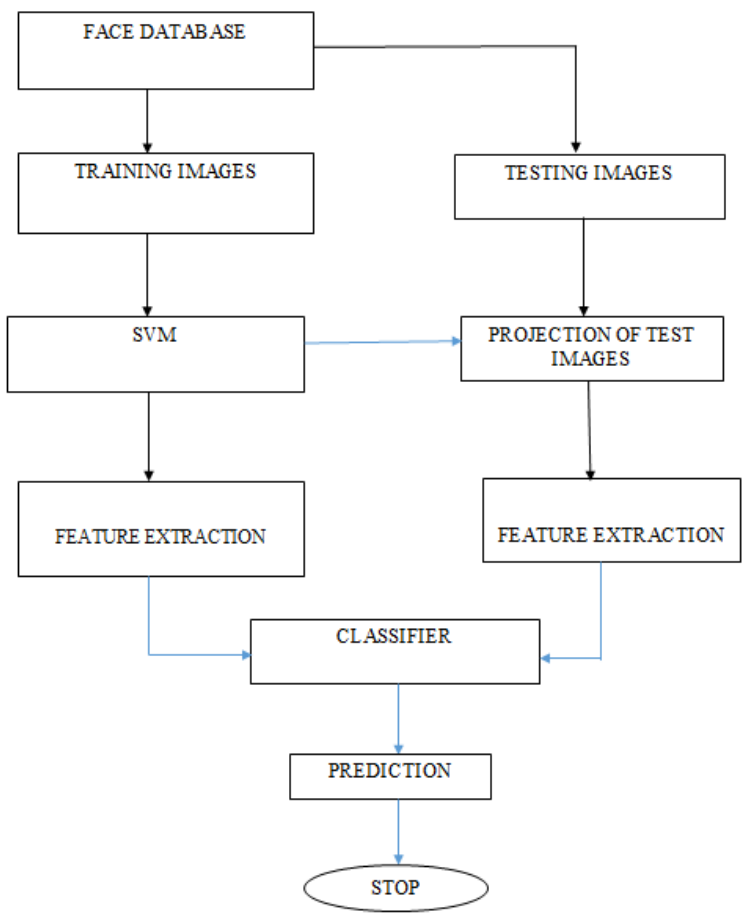

Figure 1: Overview of the Proposed System 


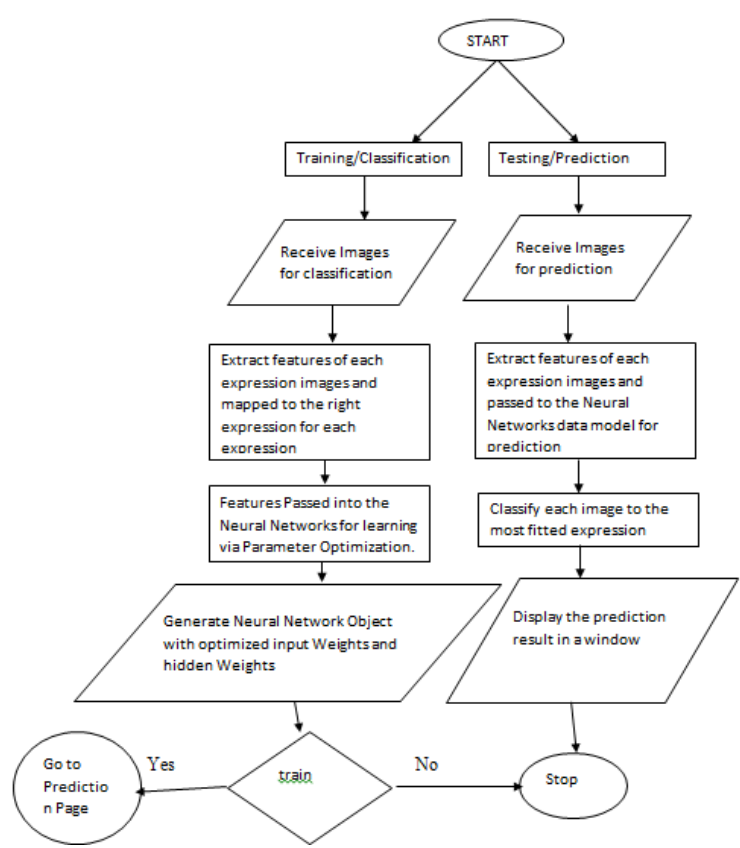

Figure 2: Flow Chart of the System

\section{METHODOLOGY}

The system was developed after the model has been designed, the system is designed to recognize three basic expressions only which are happy, sad and angry. The learning algorithm for the model deployed is Neural Networks with one hidden perceptron's for Parameter Optimization, it is a supervised learning model[6][7][11]\& [16].

Every image is received as jpeg files, Images are then converted to buffered images (object of a class in java) for graphical feature extractions. Feature extraction on each images received is being extracted using principal component differences and dimensions, extracted features are passed to the neural networks[6], [7], [15] \& [18]. Classification function of each class is given as and the classification is modeled by winner takes all i.e. class with highest probability owns data. Java object oriented programming language and support vector machines (SVM) algorithm was used in the design and implementation of this system[4]. The system has four interfaces and they are;

- $\quad$ Home page

- $\quad$ Training phase

- Testing phase

- Prediction and output result

\subsection{The Home Page}

The home page is the first interface of the system, it is the first page where users can click on file and then select option on what they want to do on the system. The file button has different options which are classification, predict, view result and exit. Classification takes users to the training phase, predictions shows an interface for testing data and prediction of the result view result brings out the output result after prediction.

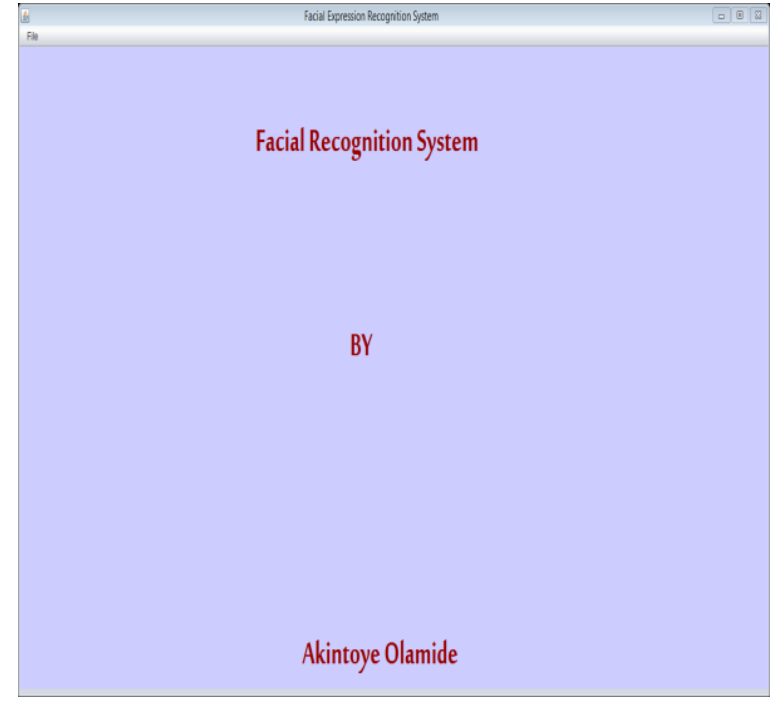

Figure 3: The Home Page

\subsection{Training Phase}

During training, the network is trained to associate outputs with input patterns. When the network is trained, it identifies the input pattern and tries to output the associated output pattern. The power of neural networks is realized when a pattern of tokens, during testing, is given as an input and it identifies the matching pattern it has already learned during training.

The User will browse on the data for the three different expressions, select a file name for the data model and then click on the train button. The process is then completed and moves to the testing phase. Click on the browse button for happy face and select an image that will be used to train the system, the same procedure is done for the other two expressions which are happiness and sadness. When this is done, the user then clicks on the train button and accuracy result comes up. The result is then completed and moves on to the testing phases to test the image that is to be predicted. The data model file that was used in the testing phase is stored in a folder where the software is located as a .ser file

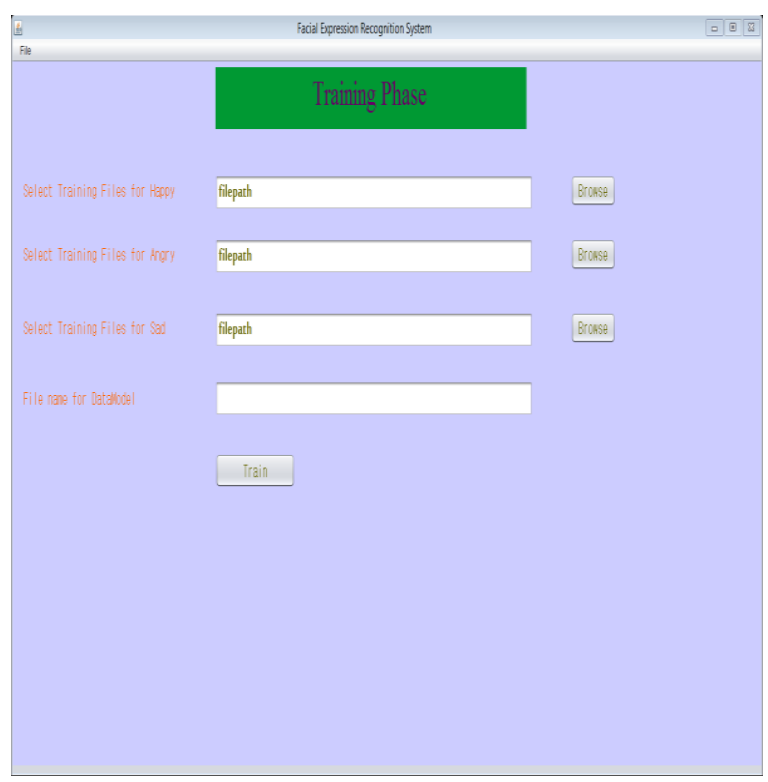

Figure 4: Training Phase without Data 


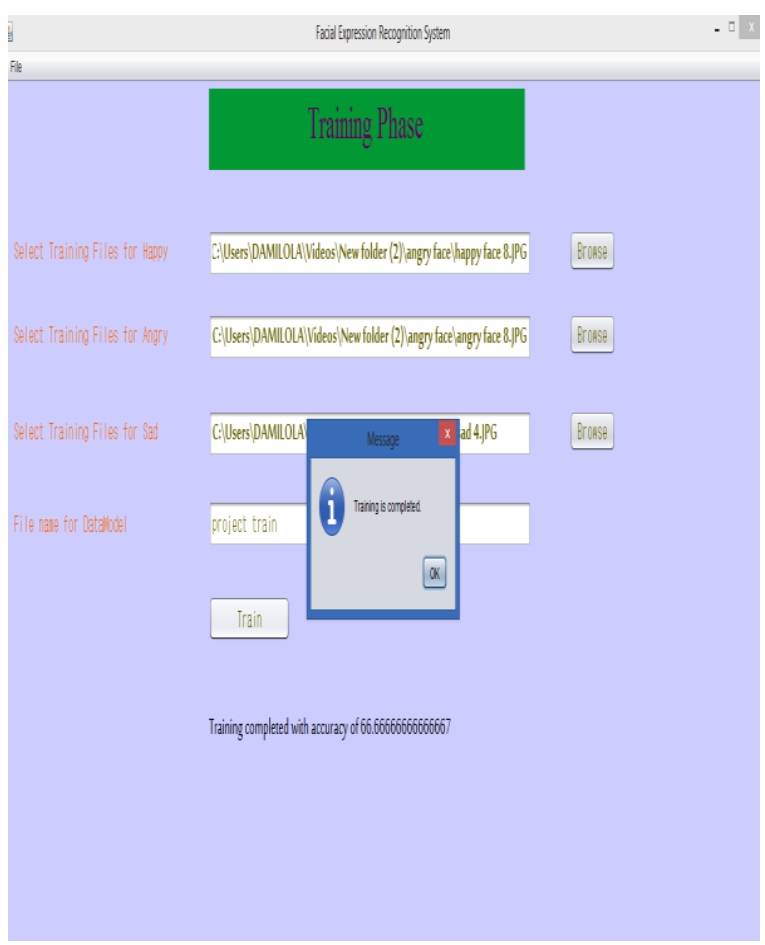

Figure 5: Training Phase with Data

\subsection{Testing phase}

Once the training is over, the system is ready to recognize gesture presented as its input. If user wants to recognize the gesture of existing image, then it can be loaded from memory of the computer and the data model file is select from the folder where it has been saved during the training phase in a .ser format. To test the system, three different sets of gestures of persons are prepared. These images are totally different from the learning subset of images in the sense that each face image was taken at different time with different instance of gesture. The system was trained using the 3 different gesture images. The category used for the training and testing are angry faces in table 1 , sad faces table 2 , and happy faces in table 3. After the data has been inputted, the user select a filename for the prediction result and click on the predict button. A message box pops up to indicate that testing of that image data has been completed successfully.

Table 1: Angry Faces

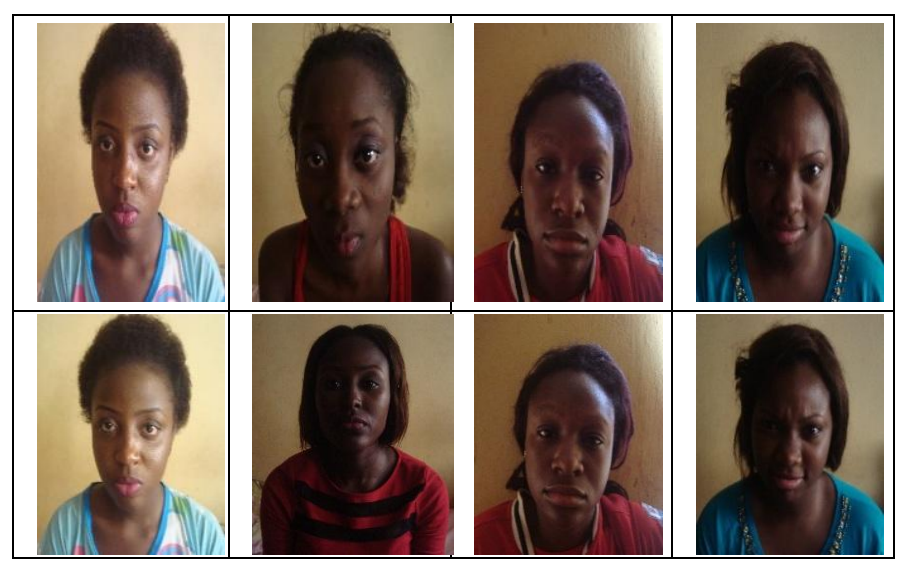

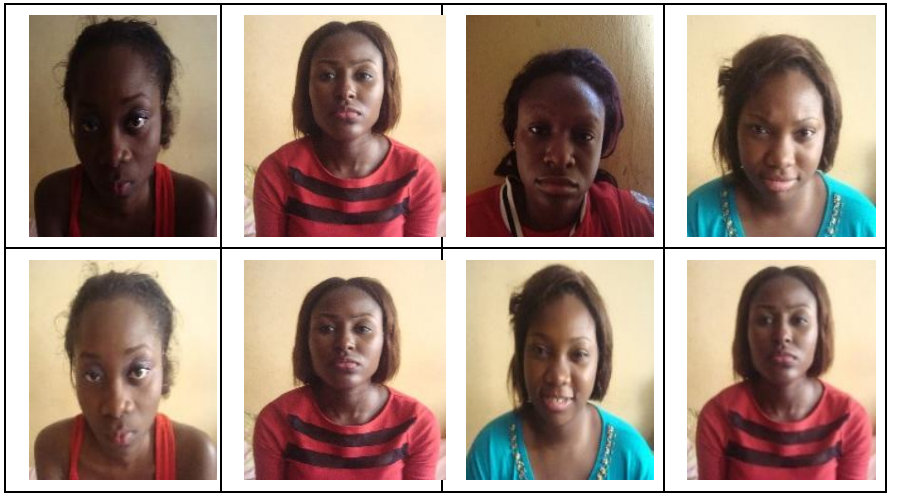

Table 2: Sad Faces

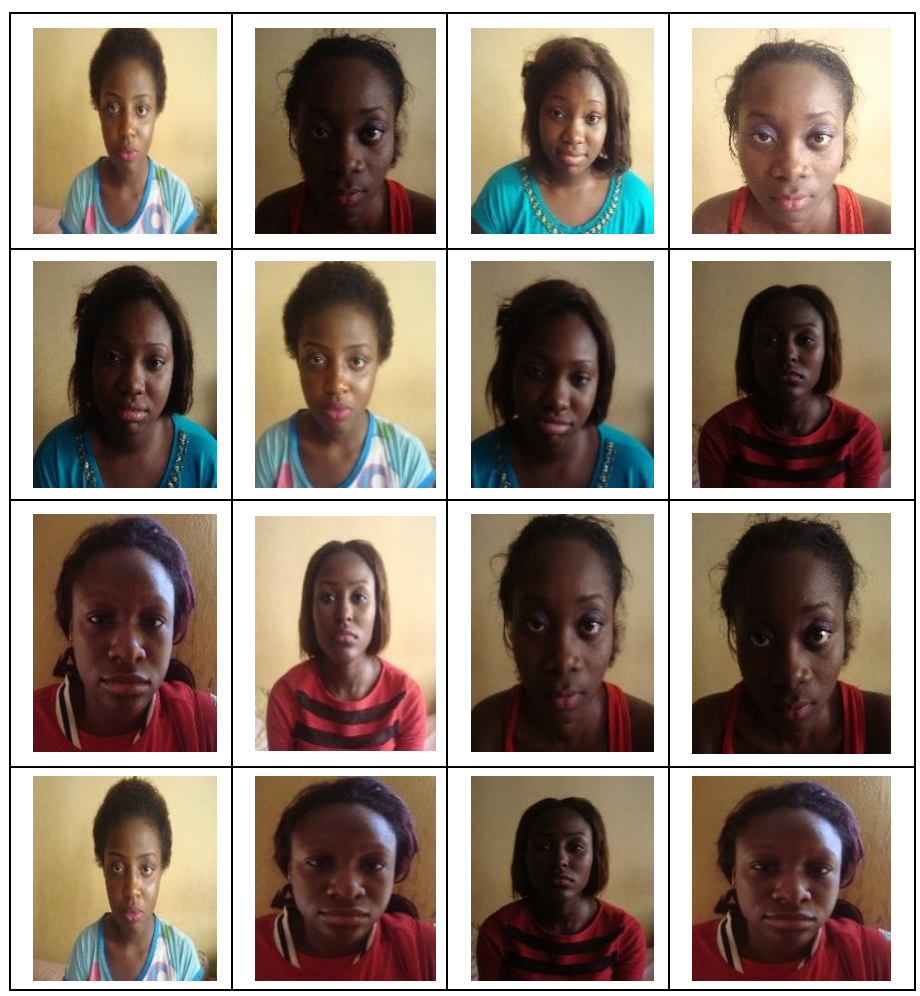

Table 3: Happy Faces

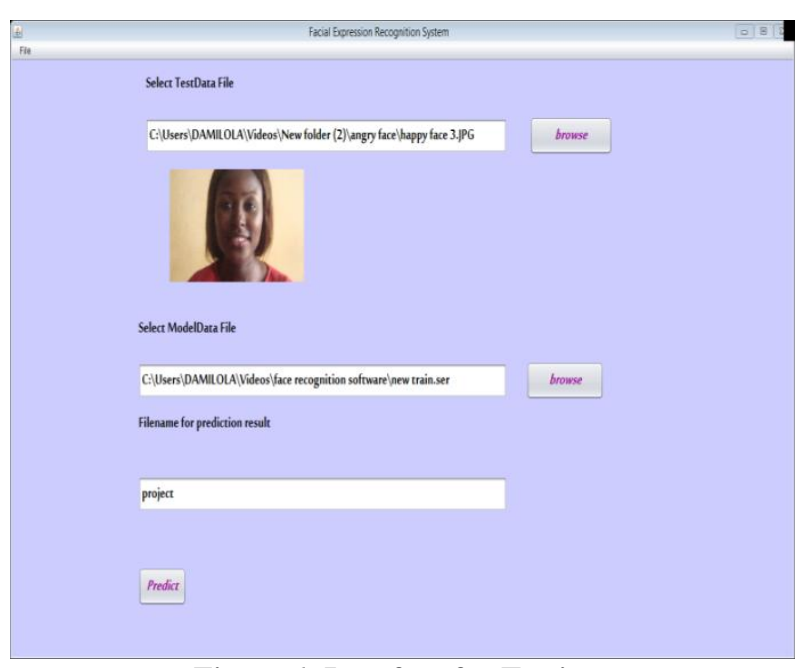

Figure 6: Interface for Testing 


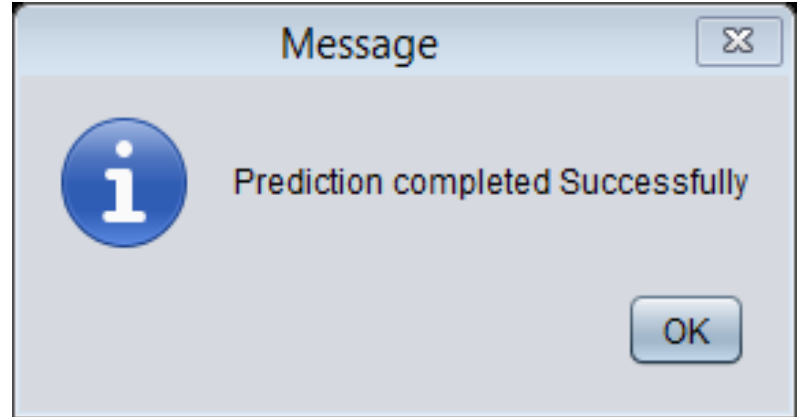

Figure 7: Testing Message

\subsection{Prediction of Result}

This phase shows the predicted result of the data that has been tested, it shows if the data that was tested is in a happy, sad or angry phase. During prediction, a word document that was saved as the file name opens and shows data of the result and prediction. This word document is also saved in the folder where software is located. Figure 7 below is an example of the output result of multiple data

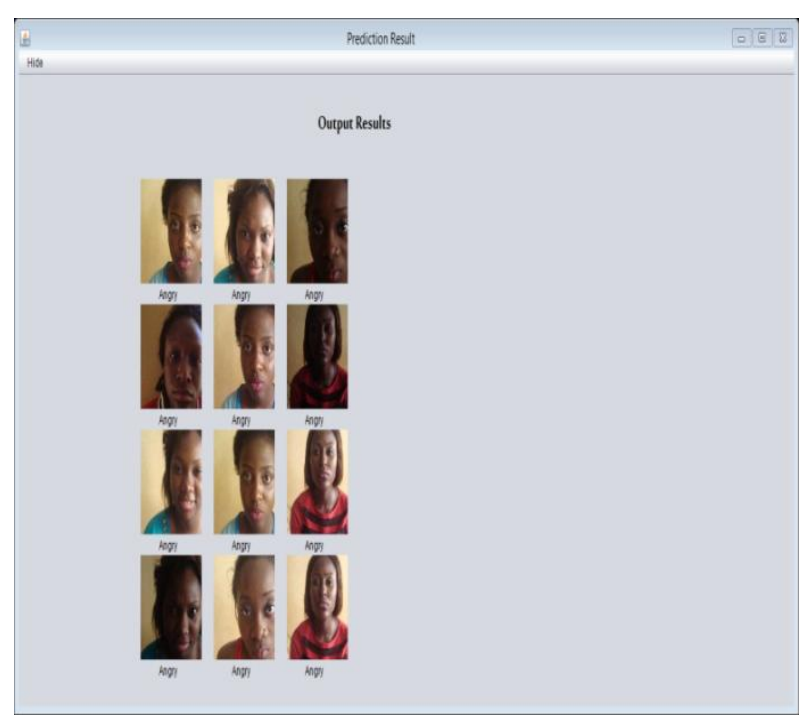

Figure 8: Output Result for Multiple Data

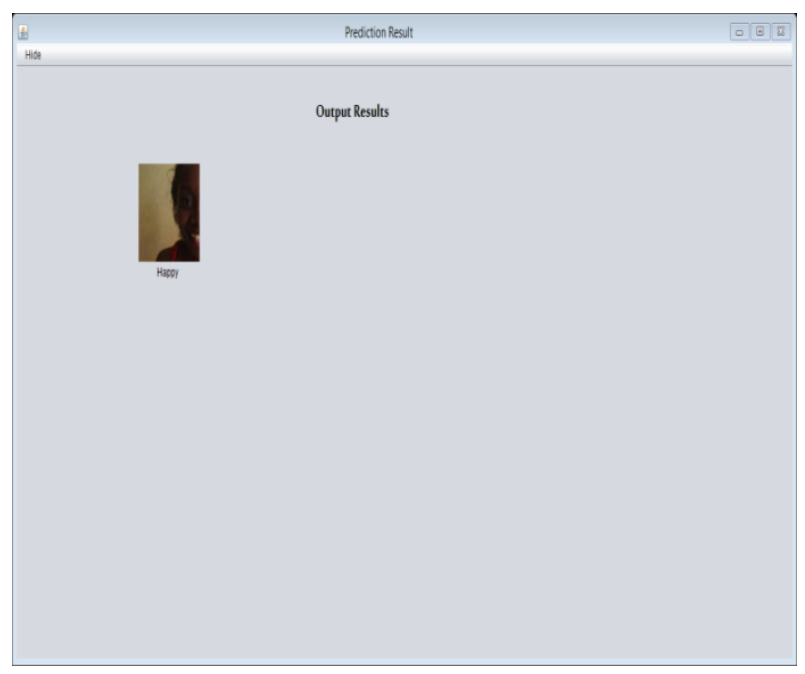

Figure 9: Output Result for Single Data

The table below show the statistical summary of the result gotten when the images were tested with the system.
Table 4: Statistical Summary of the Result

\begin{tabular}{|l|l|l|l|}
\hline $\begin{array}{c}\text { No. of } \\
\text { Input } \\
\text { Images }\end{array}$ & $\begin{array}{c}\text { Type of } \\
\text { Gesture }\end{array}$ & Recognized & $\begin{array}{c}\text { Results } \\
(\%)\end{array}$ \\
\hline 16 & ANGRY & 12 & $75 \%$ \\
\hline 16 & SAD & 9 & $60 \%$ \\
\hline 16 & HAPPY & 13 & $81.25 \%$ \\
\hline
\end{tabular}

\section{SYSTEM REQUIREMENTS}

Hardware requirements

The hardware required for development of a facial expression detecting system can work on any compatible personal computer with the following properties;

- Pentium Processor $600 \mathrm{MHz}$ minimum (1 GHz recommended)

- $\quad 512 \mathrm{MB}$ of RAM minimum (1GB recommended)

- Up to 2.5GB of Hard Disk space may be needed

- A color VGA or SVGA monitor.

- A simple enhanced windows keyboard.

- Screen Resolution 800x600 with 256 colors minimum (recommended 1024x768 in 16-bit high colors)

- $\quad$ Camera of 5MP minimum

\section{Software Requirement}

The software resources required for the developed system to function effectively and efficiently are:

- Window XP/Window 7.

- JAVA: the reason why java was used for the project is because the programming language has a very user friendly interface, it is easy and flexible to use

System Restrictions and Limitations

- The system is to detect facial expression only, its scope is not in individual facial recognition or tracking.

- The learning machines are sensitive to supply data. They tend to learn wrong thing from wrong data set. And extreme outliers will influence their performances.

- Images acquisition materials: In acquisition, Capturing device must model the realistic color in their color representations. Images with manipulated colors are unpredictable in feature extractions.

- Images are expected to have just one human face on it.

- Images are to have the same size and picture type.

- The system can only detect three emotions. 
Table 3: Happy Faces

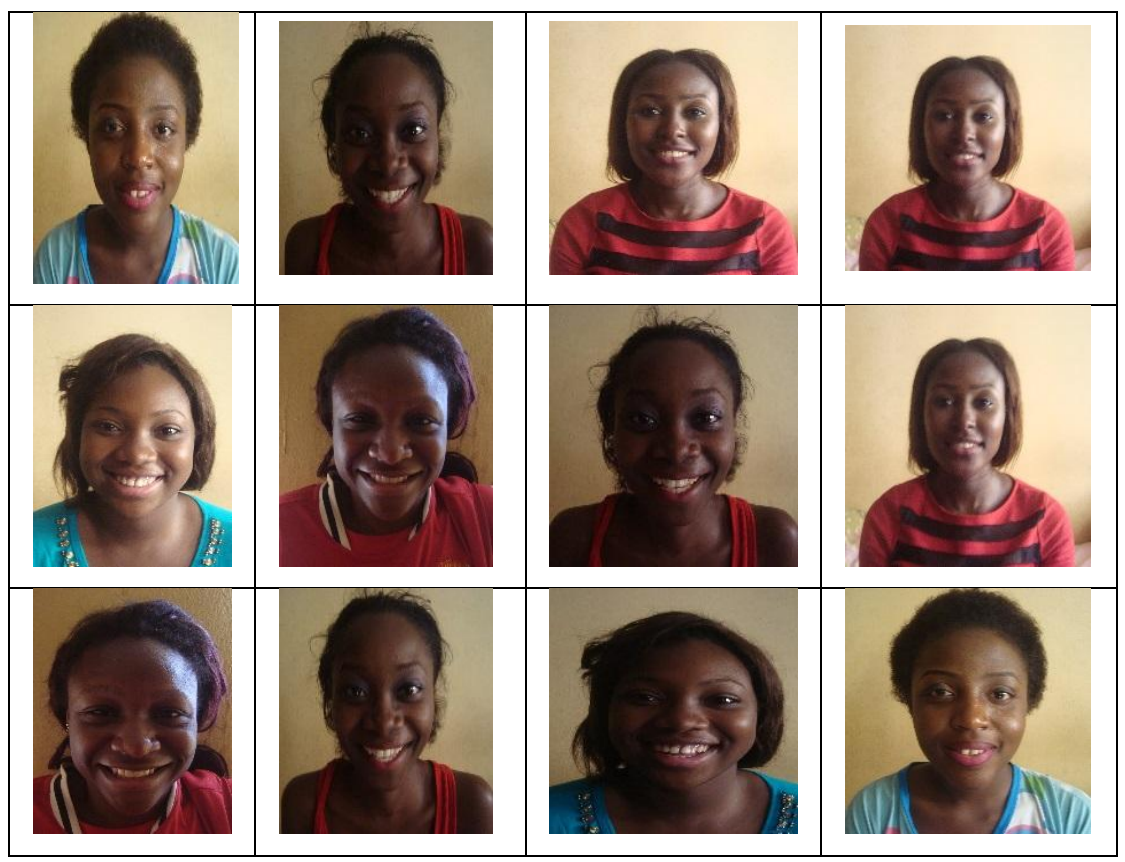

\section{REFERENCES}

[1] Abdulrahman, Muzammil, Tajuddeen R.Gwadabe, Fahad J. Abdu, and Alaa Eleyan. "Gabor wavelet transform based facial expression recognition using PCA and LBP." In Signal Processing and Communications Applications Conference, 2014 22nd, pp. 2265-2268. IEEE, 2014

[2] Baskerville, R. (1991). "Risk Analysis as a Source of Professional Knowledge". Computers \& Security 10

[3] Boehm. B (2000), "Spiral Development: Experience, Principles, and Refinements"

[4] Cortes C. Vapnik V. (1995). "Support-vector networks". Machine Learning 20

[5] Ekman. P and Friesen. W (1978) "Facial Action Coding System: A Technique for the Measurement of Facial Movement"

[6] Henry A. Rowley, Shumeet Baluja \&Takeo Kanade. (1997) Rotation Invariant Neural Network-Based Face Detection, December, CMU-CS-97-201

[7] Henry Rowley, Baluja S. \& Kanade T. (1999) "Neural Network-Based Face Detection, Computer Vision and Pattern Recognition", Neural Network-Based Face Detection, Pitts-burgh, Carnegie Mellon University, PhD thesis.

[8] International Journal of Computer Trends and Technology (IJCTT) - volume 17 Number 4 Nov 2014 Page 161

[9] International Journal of Application or Innovation in Engineering \& Management (IJAIEM) Volume 2, Issue 6, June 2013

[10] Jaimini Suthar, Narendra Limbad "A Literature Survey on Facial Expression Recognition techniques using Appearance based features"
[11] Jyh-Yeong Chang and Jia-Lin Chen (2001) "Automated Facial Expression Recognition System Using Neural Networks"

[12] KahKay Sung \& Tomaso Poggio (1994) Example Based Learning For View Based Human Face Detection, Massachusetts Institute of Technology Artificial Intelligence Laboratory and Center For Biological And Computational Learning, Memo 1521, CBCL Paper 112, MIT, December

[13] Lonnie D. Bentley p. "Systems Analysis and Design for the Global Enterprise".

[14] L. Beaurepaire, K.Chehdi, B.Vozel (1997), "Identification of the nature of the noise and estimation of its statistical parameters by analysis of local histograms", Proceedings of ICASSP-97, Munich, 1997.

[15] Manal Abdullah1, Majda Wazzan1 and Sahar Bo-saeed "Optimizing Face Recognition Using PCA" International Journal of Artificial Intelligence \& Applications (IJAIA), Vol.3, No.2, March 2012.

[16] Marian Beszedes \& Milos Oravec (2005) “A System For Localization Of Human Faces In Images Using Neural Networks", Journal Of Electrical Engineering, Vol. 56, No 7-8, pp195-199.

[17] Marakas, James A. O'Brien, George M. (2010). Management information systems.

[18] Meher, Sukanya Sagarika, and Pallavi Maben "Face recognition and facial expression identification using PCA." In Advance Computing Conference, 2014 IEEE International, pp. 1093- 1098. IEEE, 2014.

[19] Ding, W. and Marchionini, G. 1997 A Study on Video Browsing Strategies. Technical Report. University of Maryland at College Park.

[20] Fröhlich, B. and Plate, J. 2000. The cubic mouse: a new device for three-dimensional input. In Proceedings of the 
SIGCHI Conference on Human Factors in Computing Systems

[21] Tavel, P. 2007 Modeling and Simulation Design. AK Peters Ltd.

[22] Sannella, M. J. 1994 Constraint Satisfaction and Debugging for Interactive User Interfaces. Doctoral Thesis. UMI Order Number: UMI Order No. GAX9509398., University of Washington.

[23] Forman, G. 2003. An extensive empirical study of feature selection metrics for text classification. J. Mach. Learn. Res. 3 (Mar. 2003), 1289-1305.

[24] Brown, L. D., Hua, H., and Gao, C. 2003. A widget framework for augmented interaction in SCAPE.

[25] Y.T. Yu, M.F. Lau, "A comparison of MC/DC,
MUMCUT and several other coverage criteria for logical decisions", Journal of Systems and Software, 2005, in press.

[26] Spector, A. Z. 1989. Achieving application requirements. In Distributed Systems, S. Mullender

[27] Mu-Chunsu and Chien-Hsing Chou "Application of Associative memory in human face detection

[28] Samiksha Agrawal, Pailavikhatri and Shashikant Gupta (2014). "Facial expression recognition technique: A survey.

[29] Zermir Narima, Ramdani Messaond, Saaidia. M, Snani Cherifa (2015). "two dimensional principal component Analysis (2DPCA) for human facial expression recognition. 